\title{
Direct Measurement of the Nanoscale Mechanical Properties of NiTi Shape Memory Alloy
}

Gordon A. Shaw ${ }^{1}$, Wendy C. Crone ${ }^{2}$

${ }^{1}$ Department of Chemistry, University of Wisconsin-Madison

Madison, WI 53706, U.S.A.

${ }^{2}$ Department of Engineering Physics, University of Wisconsin-Madison

Madison, WI 53706, U.S.A.

\begin{abstract}
The mechanical properties of sputter-deposited NiTi shape memory alloy thin films ranging in thickness from $35 \mathrm{~nm}$ to $10 \mu \mathrm{m}$ were examined using nanoindentation and atomic force microscopy (AFM). Indents made in films as thin as $150 \mathrm{~nm}$ showed partial shape recovery upon heating, although film thickness was found to have a marked effect on the results. A modified spherical cavity model is used to describe the findings, which suggest that the substrate tends block the shape memory effect as film thickness decreases below a threshold level which is specific to applied load. This has the net effect of decreasing the indent recovery below the critical film thickness. The fact that the spherical cavity model predicts the critical film thickness at which the shape memory effect is blocked indicates that the increased recovery of nanoscale indentations is due to a suppression of plastic processes rather than an enhancement of shape memory processes.
\end{abstract}

\section{INTRODUCTION}

NiTi shape memory alloy (SMA) is a unique material that combines resilience, good biocompatibility, and suitability for thin film deposition with a fascinating wealth of chemical and physical phenomena. Chief among these is a solid state phase change known as a martensitic transformation which allows for the shape memory effect (SME). As a result, it has been the subject of a great deal of study [1]. Nevertheless, significant questions about the complex behavior of this alloy remain. Among the unanswered questions is how its mechanical properties change as the size scale considered changes from micrometers to nanometers.

Various methods have been used to probe the mechanical properties of NiTi, including uniaxial tensile and compressive loading, and microindentation [2,3]. Nanoindentation is well suited for the testing of very small volumes of material. In this technique, a load is applied to a diamond probe, which penetrates the surface of the material being tested. Analysis of the load-displacement curves then yields information about the material, particularly the Young's modulus, and hardness [4]. Indentation shape recovery has also been demonstrated on the microscale [5]. Our nanoscale work shows good agreement with the microscale data, and also extends the measurements to thin film materials [6] as well as different kinds of deformation (nanoscratch and nanowear) [7]. It also indicates that shape recovery increases markedly at indentation depths less than $100 \mathrm{~nm}$. Factors influencing pseudoelastic phenomena at this size scale 
have also been investigated [8]. The present study was performed to examine the micro and nanoscale properties of NiTi SMA by examining the effect of film thickness on the shape memory properties of NiTi.

\section{EXPERIMENTAL}

NiTi thin films were deposited at TiNi Alloy Co., San Leandro, CA. DC magnetron sputtering was used to coat substrates of oxidized Si to depths of approximately $10,1.7,0.7,0.35,0.15,0.075$ and $0.035 \mu \mathrm{m}$ with Nitinol (roughly 49.5 atomic $\% \mathrm{Ni}$ ). Subsequent to annealing, the 10 and $1.7 \mu \mathrm{m}$ films were found to be martensite by x-ray diffraction, and the $0.15 \mu \mathrm{m}$ film was determined to be a mixture of austenite and $\mathrm{R}$ phase by TEM.

The indentation experiments in this study were performed using a Digital Instruments Multi mode AFM in conjunction with a Hysitron Triboscope nanoindenter. The apparatus was set up such that the diamond tip used for indentation could be used to image the sample by using the AFM scanner. This configuration also allowed precise positioning of the indenter tip to within several $\mathrm{nm}$ of the desired indentation area. A diamond Berkovich tip was used for indentation.

Four indents were made at both 500 and $250 \mu \mathrm{N}$ loads within a $100 \mu \mathrm{m}^{2}$ area. The surface was then imaged and heated to $90 \mathrm{C}$ in situ with a Peltier cell, allowing transformation to occur by the shape memory effect. After cooling, the indents were imaged again. The change in the depth of the remnant indents due to the shape memory effect was quantified using a profiling feature of the Digital Instruments software.

\section{RESULTS}

AFM images of a $0.5 \mathrm{mN}$ nanoindent in the $700 \mathrm{~nm}$ film are shown in figure 1 before and after being heated past the transformation temperature. The indent becomes shallower as a result of the heating, which indicates recovery of the deformation accommodated through shape memory processes. This recovery can be quantified using a recovery ratio

recovery ratio $=\frac{D_{b h}-D_{a h}}{D_{b h}}$,

where $D_{b h}$ and $D_{a h}$ are indent depth before and after heating, respectively

The insets in figure 1 show profiles of the indentations before and after heating which were used to determine $D_{b h}$ and $D_{a h}$, respectively. Profiles were taken along a line from the indent's deepest point to the vertex of the triangular impression to ensure the same section was being profiled. It should be noted that there may be significant tip broadening present as a result of imaging with the relatively blunt Berkovich tip, although this does not have a significant impact on the measured recovery ratio. The indents on the 1.7 and $10 \mu \mathrm{m}$ film were imaged with a sharper AFM cantilever tip and show comparable results to those from the images obtained using the Berkovich tip. 

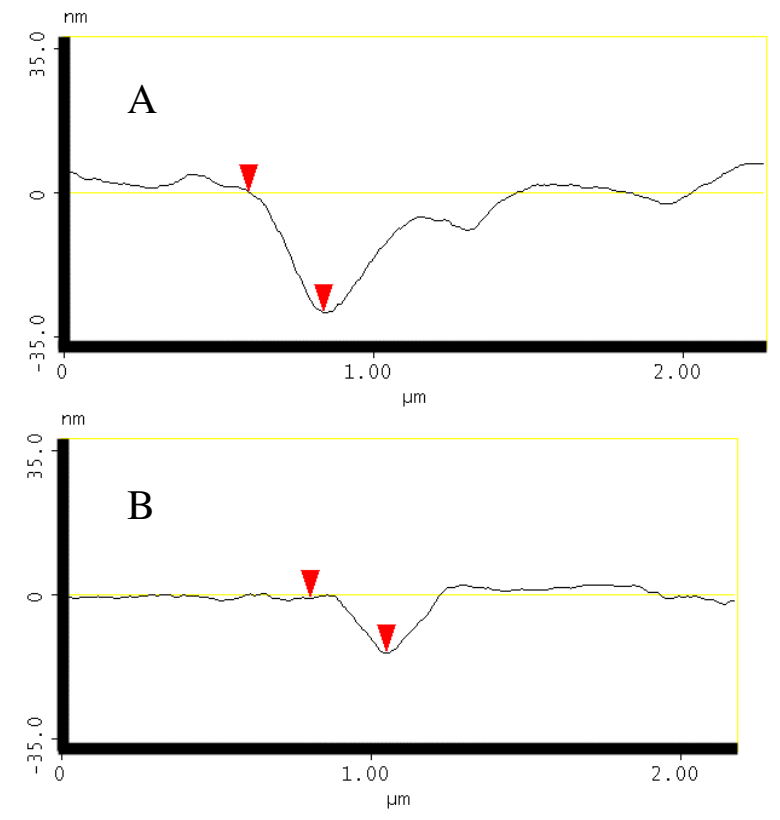
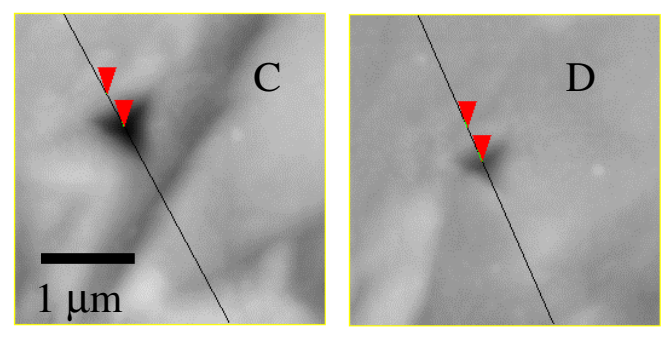

Figure 1. Profiles of a $0.5 \mathrm{mN}$ indent made on a $700 \mathrm{~nm}$ NiTi SMA film before (A) and after (B) heating. AFM images $\mathrm{C}$ and $\mathrm{D}$ show the indent topography before and after shape recovery, respectively.

Figure 2 shows recovery ratio as a function of film thickness. The recovery ratio decreases markedly at film thickness less than $350 \mathrm{~nm}$ for both the 0.25 and $0.5 \mathrm{mN}$ loads. The recovery ratio of the $710 \mathrm{~nm}$ film depended on applied load. While the 0.25 $\mathrm{mN}$ indent showed the same recovery as that of the $10 \mu \mathrm{m}$ film, the $0.5 \mathrm{mN}$ indent recovered significantly less. No discernable indentation recovery was observed in films of thickness $70 \mathrm{~nm}$ and below, or for a $1.7 \mu \mathrm{m}$ film of amorphous NiTi.

\section{DISCUSSION}

In order to understand the shape memory effect during indentation, it is necessary to develop a picture of the processes occurring under the indenter tip as it applies a load to the surface being studied. Deformation of the solid can occur by plastic deformation, martensite twin rearrangement or transformation, and elastic deformation. In the region nearest the indenter tip, stresses will be greatest and will decrease further away from the tip. So, the indentation process for SMAs can be described by a series of concentric shells. The innermost shell is in the highest stress state, and will plastically deform. Further from the indenter tip, at a slightly lower stress, deformation will occur predominantly by martensite twin rearrangement or transformation. Still further out, the stress will be even lower and can be accommodated by elastic deformation. Although it is likely that these regions are not sharply delineated, but rather blend together more continuously, a modified spherical cavity model can be used to locate the plastic/shape memory boundary, and shape memory/elastic boundaries radius within the solid [9] such that

$c=\frac{d}{\tan \beta}\left[\frac{E \tan \beta}{6 Y(1-v)}+\frac{2-4 v}{3-3 v}\right]^{1 / 3}$, 
where $\mathrm{c}$ is an elastic-plastic boundary, $\mathrm{d}$ is indentation depth, $\beta$ is indenter half angle $\left(14^{\circ}\right.$ for a Berkovich indenter), E is Young's modulus, $\mathrm{Y}$ is yield stress of the material, and $v$ is Poisson's ratio ( 0.4 for NiTi).

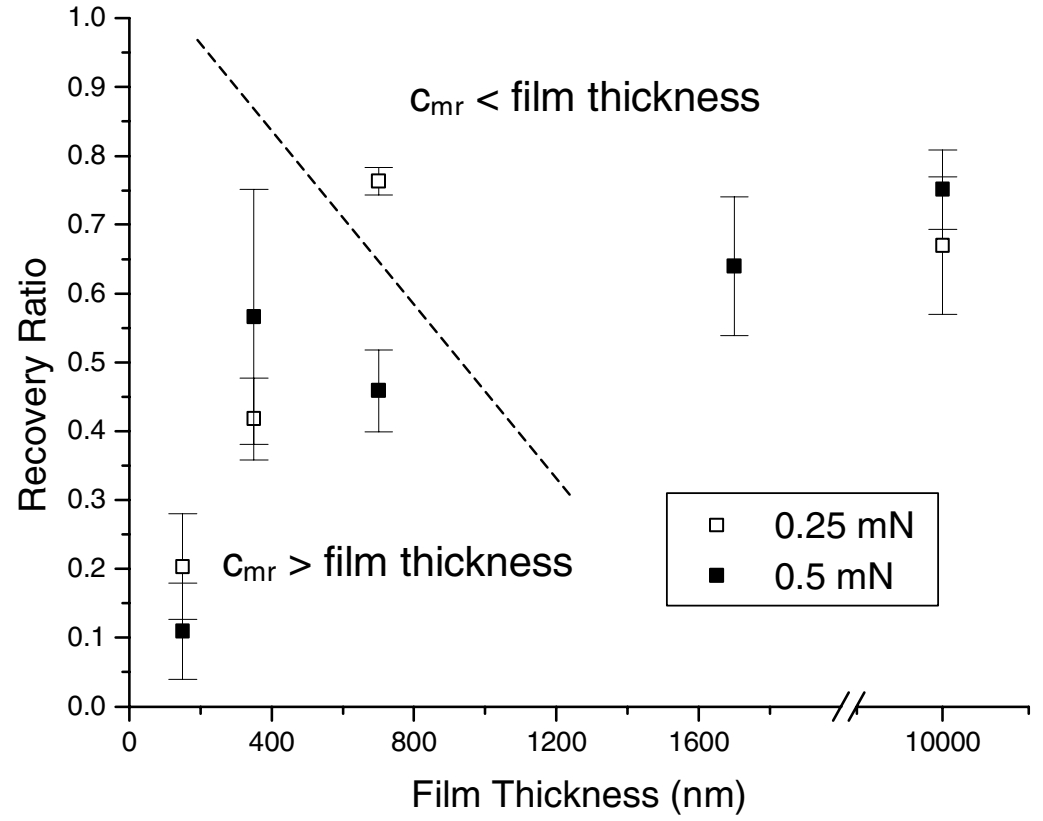

Figure 2. The variation in recovery ratio for different SMA film thicknesses. The dashed line indicates the approximate demarcation for the onset of reduction of shape memory recovery by substrate effects.

According to our model [6], the radius of the region which deforms through shape memory processes, $c_{\mathrm{mr}}$, can be estimated as a function of indent depth using $\mathrm{E}=60 \mathrm{GPa}$ [10] and $\mathrm{Y}=0.2 \mathrm{GPa}$ (the critical stress for martensite twin reorientation). Likewise, $\mathrm{c}_{\mathrm{pl}}$ can be estimated using a maximum value of $0.8 \mathrm{GPa}$ for Y [1]. Upon heating, material within the shape memory zone can recover strain induced by indentation, thereby decreasing the depth of impressions left from nanoindentation. The plastic deformation in the region bounded by $c_{\mathrm{pl}}$, however, will remain. This modified spherical cavity model suggests the recovery ratio should remain constant with indentation depth at a value less than or equal to 0.4 for a Berkovich tip geometry. This is indeed the case for indent depths greater than $100 \mathrm{~nm}$ on films thicker than $1.7 \mu \mathrm{m}$, and good agreement is seen between our data and that of Wangyang et al. for loads greater than about $3 \mathrm{mN}[5,6]$.

To elucidate the effects of constraining the dimensions of shape memory alloys, indentation recovery was examined for thinner films. Figure 3 illustrates the progressive onset of substrate effects for two fixed loads of 0.25 and $0.5 \mathrm{mN}$ as film thickness decreases. The approach used was to calculate the radius of the shape memory zone, $\mathrm{c}_{\mathrm{mr}}$, using equation 2 , normalized to the film thickness as a function of the maximum indent depth measured when the indenter was fully loaded. In the case where $\mathrm{c}_{\mathrm{mr}}<$ film thickness, no substrate effects are expected. As $\mathrm{c}_{\mathrm{mr}}$ approaches the film thickness, substrate effects begin to occur. The maximum indentation depth at a given load normally remains constant for a bulk material, as is the case when $\mathrm{c}_{\mathrm{mr}} /$ film thickness is 
less than one. The hardness of $\mathrm{Si}$ is about $12 \mathrm{GPa}$, compared to $3 \mathrm{GPa}$ for NiTi [10]. A Si substrate would be expected to resist deformation much more than NiTi, thereby decreasing the maximum indent depth at a given load relative to bulk NiTi. This is indeed the case when $\mathrm{c}_{\mathrm{mr}}$ / film thickness is greater than 1 .

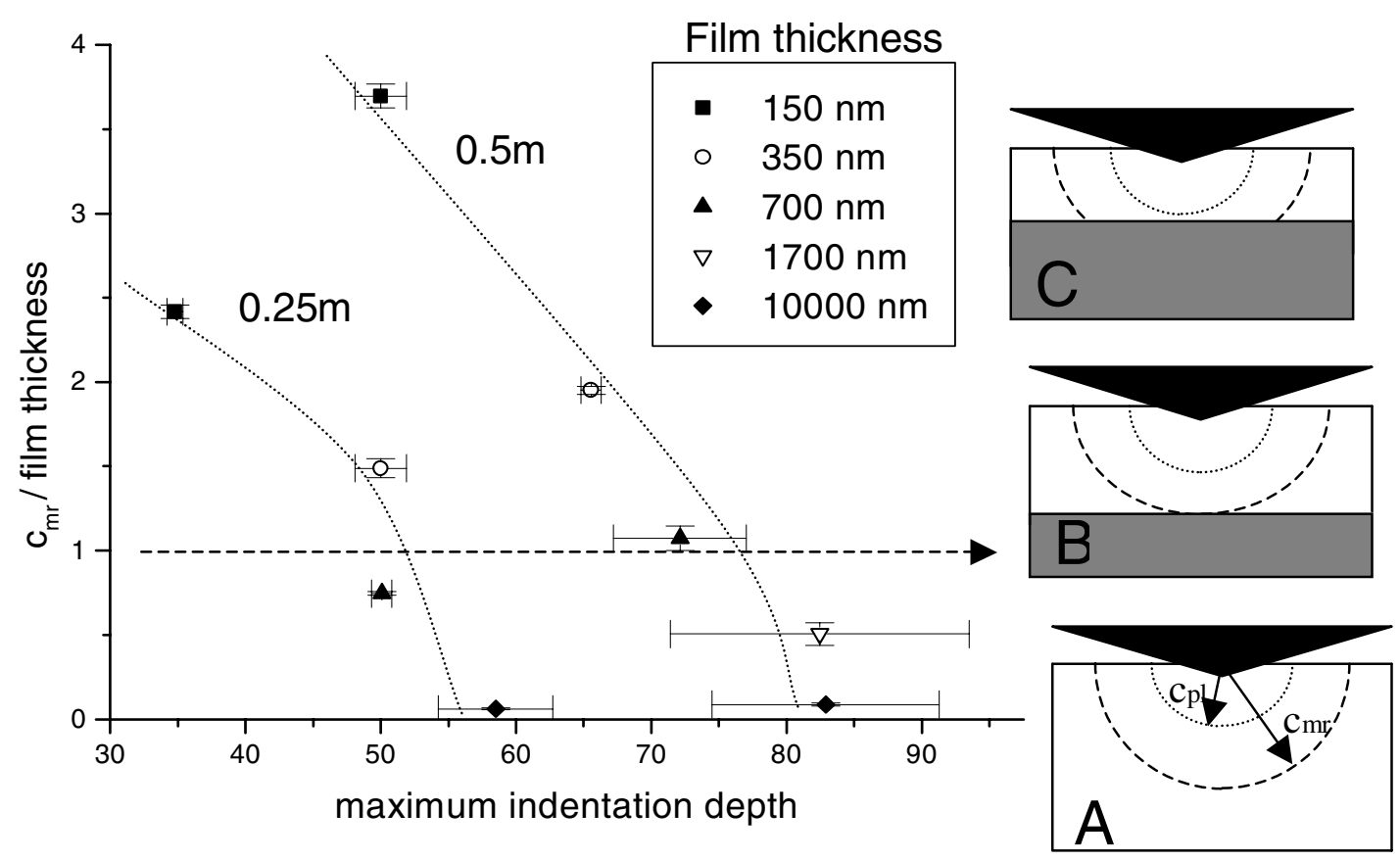

Figure 3. The relationship of the spherical cavity model to the onset of substrate effects for an SMA. The graph shows the ratio of $\mathrm{c}_{\mathrm{mr}}$ calculated using equation 2 to film thickness vs. maximum indentation depth at 0.25 and $0.5 \mathrm{mN}$ loads for the film thicknesses indicated in the graph's legend. Diagram A illustrates the spherical cavity model in the absence of substrate effects $\left(\mathrm{c}_{\mathrm{mr}}<\right.$ film thickness). In this model, the radius of the hemispherical boundary within which plastic deformation occurs is $\mathrm{c}_{\mathrm{pl}}$ and the radius within which recoverable deformation occurs by processes related the the SME is $\mathrm{c}_{\mathrm{mr}}$. Diagram B shows the onset of substrate effects $\left(c_{\mathrm{mr}}=\right.$ film thickness). Diagram $\mathrm{C}$ illustrates behavior which is clearly influenced by substrate effects ( $c_{\mathrm{mr}}>$ film thickness). The curves describing behavior at different loads are presented as guides to the eye.

The data in figure 2 show that film thickness can have a marked effect on indent shape recovery. Since the Si substrate cannot accommodate significant reversible deformation, one would expect the substrate to constrain the shape memory deformation. As the shape memory zone impinges on the substrate, the deformation can no longer be accommodated by shape memory processes, and a decrease in recovery ratio is observed. As an example, consider the data for the $700 \mathrm{~nm}$ film. Figure 3 illustrates that the shape memory zone has not yet impinged on the substrate surface at a load of $0.25 \mathrm{mN}$ (i.e. $\mathrm{c}_{\mathrm{mr}} /$ film thickness $<1$ ), but has for the $0.5 \mathrm{mN}$ load at this film thickness. This translates clearly to a quantifiable difference in recovery ratio in figure 2 where the $0.5 \mathrm{mN}$ load recovers significantly less deformation in the $700 \mathrm{~nm}$ film. 
Since the radius of the shape memory zone appears to follow the spherical cavity predictions regardless of size scale, this suggests that the increase in shape memory recovery at the nanoscale is the result of a decrease in the occurrence of plasticity rather than an enhancement of the shape memory effect. This may partly explain the origin of the increased recovery of indents less than $100 \mathrm{~nm}$ deep observed in a previous study [6].

\section{CONCLUSIONS}

The shape memory effect has been shown to occur on the nanoscale for NiTi films as thin as $150 \mathrm{~nm}$ which have been deformed by nanoindentation. The onset of substrate effects can be predicted using a modified spherical cavity model. For thinner films, indent recovery was inhibited as the region in which the shape memory effect occurs impinged on the silicon substrate. This study indicates that previously observed enhancement of recovery for nanoscale indentations results from the inhibition of plasticity at small length scales.

\section{ACKNOWLEDGEMENTS}

The authors would like to thank Dr. A. David Johnson of TiNi Alloy Co, San Leandro, CA for providing thin film samples, and Dr. Arthur B. Ellis for helpful discussions. This research was supported by the U.S. Department of Energy (award \#DE-FC36-01G011055).

\section{REFERENCES}

1. Otsuka, K.; Wayman, C. M. Shape Memory Materials; Cambridge University Press: Cambridge, 1999.

2. Gall, K.; Juntunen, K.; Maier, H. J.; Sehitoglu, H.; Chumlyakov, Y. I., Instrumented microindentation of NiTi SMAs. Acta. Mater., 493205-3217,2001.

3. Gall, K.; Dunn, M. L.; Liu, Y.; Labossiere, P.; Sehitoglu, H.; Chumlyakov, Y. I., Micro and macro deformation of single crystal NiTi. J. Eng. Mat. Tech, 124238-245,2002.

4. Oliver, W. C.; Pharr, G. M., An improved technique for determining hardness and elastic modulus using load and displacement sensing indentation experiments. Journal of Materials Research, 7(6), pp. 1564-1583,1992.

5. Wangyang, N.; Cheng, Y.; Grummon, D., Recovery of Microindents in a Nickel-Titanium SMA... App. Phys. Lett, 80(18), pp. 3310-3312,2002.

6. Shaw, G. A.; Stone, D. S.; Johnson, A. D.; Ellis, A. B.; Crone, W. C., Shape memory effect in nanoindentation of nickel-titanium thin films. Applied Physics Letters, 83(2), pp. 257-259,2003.

7. Crone, W. C.; Shaw, G. A.; Stone, D. S.; Johnson, A. D.; Ellis, A. B., Shape Recovery after Nanoindentation of NiTi Thin Films. Proceedings of the SEM Annual Conference on Experimental Mechanics, 1271-6,2003.

8. Ma, X.-G.; Komvopoulos, K., Nanoscale pseudoelastic behavior of indented titanium-nickel films. Applied Physics Letters, 83(18), pp. 3773-3775,2003.

9. Johnson, K. L. Contact Mechanics; Cambridge University Press: Cambridge, 1994, p. 175.

10. Fu, Y.; Huang, W.; Du, H.; Huang, X.; Tan, J.; Gao, X., Characterization of TiNi SMA thin films for MEMS applications. Surf. Coat. Technol., 145, 107-112,2001. 